typeset using JPSJ.sty $<$ ver.1.0b $>$

\title{
Quantum Effects of Resistance-Shunted Josephson Junctions
}

\author{
Takeo KATO and Masatoshi IMADA \\ Institute for Solid State Physics, University of Tokyo, \\ 7-22-1 Roppongi, Minato-ku, Tokyo 106-8666
}

(Received August 3, 1999)

\begin{abstract}
Thermodynamics and transport properties of resistance-shunted Josephson junctions are studied theoretically in the tight-binding limit $E_{\mathrm{C}} / E_{\mathrm{J}} \ll 1$, where $E_{\mathrm{C}}$ and $E_{\mathrm{J}}$ are a charging energy and a Josephson coupling energy respectively. Based on a phenomenological harmonic-oscillator model, weak coupling region $K=R_{\mathrm{Q}} / R \ll 1$ is analytically studied, where $R$ and $R_{\mathrm{Q}}=h /(2 e)^{2}$ are a shunted resistance and the quantum resistance. In addition to the effective bandwidth $2 \hbar \Delta_{\text {eff }}$, we find that this multi-level system genuinely has a novel crossover at lower energy $K \hbar \Delta_{\text {eff }}$ below which the density of states becomes strongly degenerate. These two energy scales control the linear DC responses, optical responses, and nonlinear $I$ - $V$ characteristics. The lower energy crossover indicates the existence of a new class of strongly-correlated phenomena beyond the framework of the Kondo problem.
\end{abstract}

KEYWORDS: Josephson junctions, dissipation, Coulomb blockade, macroscopic quantum effects, superconductorinsulator transition

\section{$\S 1 . \quad$ Introduction}

Small resistance-shunted Josephson junctions are simplest systems for the purpose of clarifying various macroscopic quantum effects such as quantum tupneling, quantum coherence, Coulomb blockade, etc. 1, 2. (4) Though quantum properties of Josephson junctions have been studied in many literatures, 4 thermodynamics and I-V characteristics have not been clarified except for some special limits. In this paper, we study specific heat and optical linear responses in the weak damping region analytically. We also conjecture the nonlinear I-V characteristics from the available results.

Classical dynamics of the Josephson junctions has been studied based on the equivalent circuits shown in

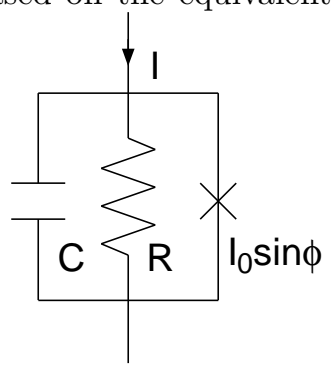

Fig. 1. The equivalent circuits for a small Josephson junction
Fig. 1. The equation of motion is described as

$$
C \dot{V}+\frac{V}{R}+I_{0} \sin \phi=I
$$

where $C, R, I_{0}, I$ are a capacitance, a shunted-resistance, a Josephson critical current, and an external current respectively. The dynamics of the phase difference $\phi$ generates a voltage $V$ across the junction through the Josephson relation

$$
V=\frac{\Phi_{0}}{2 \pi} \dot{\phi},
$$

where $\Phi_{0}=h / 2 e$ is a unit flux. From (11) and (2), the equation of motion is written as

$$
\begin{aligned}
& C \ddot{q}+\frac{1}{R} \dot{q}+\frac{\partial U}{\partial q}=0, \\
& U(q)=-I q-E_{\mathrm{J}} \cos \left(2 \pi \frac{q}{\Phi_{0}}\right),
\end{aligned}
$$

where $q=\Phi_{0} \phi / 2 \pi$ and $E_{\mathrm{J}}=\Phi_{0} I_{0} / 2 \pi$. This equation can be interpreted as an equation of motion of a dissipative particle with a coordinate $q$ in the washboard-like potential $U(q)$. The mobility $\mu$ of this dissipative particle is related to the $\mathrm{I}-\mathrm{V}$ characteristics of the junction as

$$
V=\mu I
$$

The Josephson junction has two important energy scales: one is the Josephson coupling energy $E_{\mathrm{J}}$, and the other is the charging energy $E_{\mathrm{C}}=e^{2} / 2 C$. The ratio $E_{\mathrm{J}} / E_{\mathrm{C}}$ controls the quantum effects in Josephson junctions. When the area of the junction $S$ becomes small, 
the charging energy $E_{\mathrm{C}}(\propto 1 / S)$ cannot be neglected compared with $E_{\mathrm{J}}(\propto S)$. Then, the system cannot be described by the classical equation. To study dissipative quantum systems, a phengmenological harmonicoscillator model is introduced:(1, 2)

$$
\begin{aligned}
H & =H_{0}+\sum_{j}\left\{\frac{p_{j}^{2}}{2 m_{j}}+\frac{1}{2} m_{j} \omega_{j}^{2}\left(x_{j}-\frac{c_{j}}{m_{j} \omega_{j}^{2}} q\right)^{2}\right\}(6) \\
H_{0} & =\frac{Q^{2}}{2 C}+U(q),
\end{aligned}
$$

where $Q$ is a pseudo-momentum conjugate to $q$, which corresponds to a charge of the junction. The harmonic oscillators represent an environment which generates the damping term. When the spectral function is taken as

$$
\begin{aligned}
J(\omega) & \equiv \frac{\pi}{2} \sum_{j} \frac{c_{j}^{2}}{m_{j} \omega_{j}} \delta\left(\omega-\omega_{j}\right) \\
& =\frac{\omega}{R} e^{-\omega / \omega_{\mathrm{c}}}
\end{aligned}
$$

the equation of motion (3) is reproduced in the classical limit. Here, $\omega_{\mathrm{c}}$ is a cut-off frequency.

The ground state of this model has been discussed by using perturbative renormalization analysis and duality mapping.5 The dimensionless damping strength defined by $K=R_{\mathrm{Q}} / R$ controls the ground-state properties, where $R_{\mathrm{Q}}=h / 4 e^{2}(=6.5[\mathrm{k} \Omega])$ is the quantum resistance. For $K<1$, the particle is extended in the real space. In this case, superconductivity of the Josephson junction is destroyed by large phase fluctuations, and the junction behaves as an insulator. For $K>1$, the particle is localized in a well, and the superconductivity of the Josephson junction is retained. Thus, it is predicted that there exists the superconductor-insulator transition at $K=1$. These results hayg been supported on a qualitative level by experiments.6, 6

The $I-V$ characteristics of the resistance-shunted junction have been studied in the literatures. $3,4,8,9,10,11,22,13,1$ Generally, it is difficult to study the dissipative model for arbitrary ratio $E_{\mathrm{C}} / E_{\mathrm{J}}$. In this paper, we restrict ourselves to the tight-binding limit $E_{\mathrm{C}} / E_{\mathrm{J}} \ll 1$, where the system Hamiltonian $H_{0}$ is well described by a onedimensional tight-binding model

$$
H_{0}=-\hbar \Delta \sum_{l}\left(c_{l+1}^{\dagger} c_{l}+c_{l}^{\dagger} c_{l+1}\right)
$$

in the absence of the external current. Here, $\Delta$ is a hopping amplitude, and $c_{l}\left(c_{l}^{\dagger}\right)$ is an annihilation (creation) operator at the site $l$. Even in this limit, the properties of the junction in the presence of the dissipation have been studied only for special regions in the whole parameter space. So far, there are two regions which allows analytical calculations: ope is the high-temperature or/and strong-damping limit, 11 and the other is the $K=1 / 2$ solvable line.13)
Recently, a formulation for $K \ll 1$ has been proposed by the authors 15) Based on this formulation, we can calculate thermodynamics and linear responses at any temperature. This calculation reveals the $K$-dependence of the low-temperature properties, which have not been studied so far. The calculation for the weak damping region reveals a novel feature. In the absence of dissipation, only the bandwidth $2 \hbar \Delta$ determines the characteristic energy scale of the junction. Additionally, a novel characteristic energy scale $K \hbar \Delta$ appears in the thermodynamics and transport properties. This feature can be observed most clearly in the weak damping region $K \ll 1$, and cannot be observed clearly in the $K=1 / 2$ solvable case.

In Ref. 15, the authors have treated the dissipative tight-binding model as an effective model of a dissipative carrier in solids, and have not studied applications to Josephson junctions. In this paper, by using the results of Ref. 15 and other available references, the aspects of thermodynamics and $I-V$ characteristics of Josephson junctions are presented in detail. This paper has two purposes: (1) we report comprehensive studies on the existence of the two different energy scales (especially for experimentalists), and (2) we analyze and conjecture the junction properties for general values of $K$ beyond the solvable cases.

This paper is organized as follows. The formulation of the weak damping region is summarized briefly in Sec. 2. The thermodynamics and $I-V$ characteristics are discussed in Sec. 3. In Sec. \#, the relation to the Kondo problem is discussed, and finally in Sec. 5 the results are summarized.

\section{$\S 2$. Formulation}

The partition function is formulated from (6) and (10) in terms of the path integrals as 15 .

$Z=\sum_{m=0}^{\infty} \frac{\Delta^{2 m}}{2 m !} \sum_{\left\{\xi_{l}\right\}^{\prime}} \prod_{n=1}^{2 m} \int_{0}^{\hbar \beta} \mathrm{d} \tau_{n} \exp \left[\sum_{k<l}^{2 m} \xi_{k} \xi_{l} \phi\left(\tau_{l}-\tau_{k}\right)\right]$

where $\beta=1 / k_{\mathrm{B}} T, \xi_{l}= \pm 1$, and the prime in $\left\{\xi_{l}\right\}^{\prime}$ denotes the summation in accordance with the constraint $\sum_{l} \xi_{l}=0$. The function $\phi(\tau)$ is defined by

$$
\begin{aligned}
\phi(\tau)= & \frac{\Phi_{0}^{2}}{\pi \hbar} \int_{0}^{\infty} \mathrm{d} \omega \frac{J(\omega)}{\omega^{2}}(\operatorname{coth}(\hbar \beta \omega / 2) \\
& \left.-\frac{\cosh [\omega(\hbar \beta / 2-|\tau|)]}{\sinh (\hbar \beta \omega / 2)}\right) .
\end{aligned}
$$

The expression (11) may be interpreted as a statistical model of classical interacting particles with the potential $\phi(\tau)$.

The weak coupling theory can be constructed by applying the Debye-Hïckel approximation to this classical partition function 15) It has not been proved that this 
approximation is valid for the present model. It, however, can be shown that this approximation reproduces correct results at least in the high-temperature region. It also reproduces the correct results in the continuum limit $(K \rightarrow 0, \Delta \rightarrow \infty, K \Delta=$ const.). Hence, we expect that this approximation gives correct qualitative features. The validity of the Debye-Hückel approximation is discussed in Appendix.

By the Debye-Hückelapproximation, the partition function is calculated as 15 )

$$
\begin{aligned}
& Z=\int_{0}^{2 \pi} \frac{\mathrm{d} \theta}{2 \pi} \mathrm{e}^{U(n)}, \\
& U(n)=n+\log \Gamma(K n+1)-K n \psi(K n+1), \\
& Q(n)=K(\psi(K n+1)+\bar{\gamma}), \\
& 2 \beta \hbar \Delta\left(\frac{\hbar \beta \omega_{\mathrm{c}}}{2 \pi}\right)^{-K} \cos \theta=n \mathrm{e}^{-Q(n)},
\end{aligned}
$$

where $\bar{\gamma}$ is the Euler constant, $\Gamma(x)$ is the gamma function, and $\psi(x)$ is the polygamma function. The specific heat is directly calculated from the partition function. The density of states $D(\omega)$ is also calculated from $Z(\beta)$ as

$$
Z(\beta)=\int_{\omega_{0}}^{\infty} \mathrm{d} \omega D(\omega) e^{-\hbar \beta \omega},
$$

where $\hbar \omega_{0}$ is the ground state energy.

We formulate the optical mobility $\mu(\omega)$ by the Kubo formula. The exact formal expression for $\mu(\omega)$ is given by 15 )

$$
\begin{aligned}
\mu(\omega) & =\frac{\Phi_{0}^{2}}{\hbar \omega} \operatorname{Im} \int_{0}^{\infty} \mathrm{d} t \Lambda(t) e^{\mathrm{i} \omega t}, \\
\Lambda(t) & =-2 \operatorname{Im} \tilde{\Lambda}(\tau \rightarrow \mathrm{i} t), \\
\tilde{\Lambda}(\tau) & =\frac{\Delta^{2}}{Z} \sum_{m=0}^{\infty} \sum_{\left\{\xi_{l}, \sigma, \sigma^{\prime}\right\}^{\prime}}\left(-\sigma \sigma^{\prime}\right) \frac{\Delta^{2 m}}{2 m !} \\
& \times \prod_{l=1}^{2 m} \int_{0}^{\hbar \beta} \mathrm{d} \tau_{l} \exp \left[S\left(\left\{\tau_{l}\right\}, \tau\right)\right], \\
S\left(\left\{\tau_{l}\right\}, \tau\right) & =\sum_{k<l}^{2 m} \xi_{k} \xi_{l} \phi\left(\tau_{l}-\tau_{k}\right)+\sum_{l=1}^{2 m} \sigma \xi_{l} \phi\left(\tau_{l}\right) \\
& +\sum_{l=1}^{2 m} \sigma^{\prime} \xi_{l} \phi\left(\tau_{l}-\tau\right)+\sigma \sigma^{\prime} \phi(\tau),
\end{aligned}
$$

where $\xi_{l}, \sigma, \sigma^{\prime}= \pm 1$, and the prime in $\left\{\xi_{l}, \sigma, \sigma^{\prime}\right\}^{\prime}$ denotes the summation in accordance with the constraint $\sum_{l} \xi_{l}+\sigma+\sigma^{\prime}=0$. Here, the current-current correlation function $\tilde{\Lambda}(\tau)$ can be interpreted as a classical partition function with fixed charges at $0, \tau$. Hence, the DebyeHückel approximation is also applicable in the calculation of $\tilde{\Lambda}(\tau)$ in the weak damping region $K \ll 1$. As a result, the real-time correlation function $\Lambda(t)$ is calcu- lated as

$$
\begin{aligned}
\Lambda(t) & =\frac{1}{Z} \int_{0}^{2 \pi} \frac{\mathrm{d} \theta}{2 \pi} \frac{n^{2}}{\hbar^{2} \beta^{2} \cos ^{2} \theta} e^{U(\theta)} \\
& \times\left[e^{-S(t ; \theta)}+e^{S(t ; \theta)} \cos 2 \theta\right] \sin R(t ; \theta),
\end{aligned}
$$

where $R(t ; \theta)$ and $S(t ; \theta)$ are defined as

$$
\begin{aligned}
R(t ; \theta) & =\pi K e^{-\gamma(\theta) t}, \\
S(t ; \theta) & =\frac{\pi}{\hbar \beta \gamma(\theta)}-\frac{\pi}{2} \cot \frac{\hbar \beta \gamma(\theta)}{2} e^{-\gamma(\theta) t} \\
& -\sum_{k=1}^{\infty} \frac{k \exp (-2 \pi k t / \hbar \beta)}{k^{2}-(\hbar \beta \gamma(\theta) / 2 \pi)^{2}}, \\
\gamma(\theta) & =2 \pi K n(\theta) / \hbar \beta .
\end{aligned}
$$

Thus, the linear mobility can be calculated at any frequencies.

\section{§3. Results}

In this section, we show results mainly for the weak damping region $K \ll 1$ based on the formulation of Sec. 2. In this paper, we stress the fact that the two different energy scales, $K \hbar \Delta_{\text {eff }}$ and $\hbar \Delta_{\text {eff }}$ control the junction properties. We expect that this new viewpoint is helpful to understand the junction properties for general values of $K$ and $E_{\mathrm{C}} / E_{\mathrm{J}}$.

In addition to the weak damping region $K \ll 1$, there exist some regions where analytical result can be obtained: the $K \overline{\bar{B}} \mathbf{1} / 2$ exact solution ${ }^{3}$ ) and incoherent hopping regime. 211 From these results, the thermodynamics and $I-V$ characteristics are conjectured beyond the weak damping region.

In the following subsections, we discuss the thermodynamics (Sec. 3.1), the linear DC mobility (Sec. 3.2), the linear optical mobility (Sec. 3.3), and the nonlinear DC mobility (Sec. 3.4). Finally in Sec. 3.5, the $I-V$ characteristics are derived from a simplified model, and is compared with our results.

\subsection{Thermodynamics}

The specific heat of the dissipative particle has been calculated at any temperature in Ref. 15. The low- and high-temperature form of the specific heat $C$ is calculated for $K \ll 1$ as

$$
C / k_{\mathrm{B}}= \begin{cases}4 \pi^{2}\left(\frac{2 \pi k_{\mathrm{B}} T}{\hbar \Delta_{\mathrm{eff}}}\right)^{2 K-2}, & \left(k_{\mathrm{B}} T \gg \hbar \Delta_{\mathrm{eff}}\right), \\ \frac{k_{\mathrm{B}} T}{12 K c \hbar \Delta_{\mathrm{eff}}}, & \left(k_{\mathrm{B}} T \ll \hbar \Delta_{\mathrm{eff}}\right),\end{cases}
$$

where the effective hopping amplitude is defined by $\Delta_{\text {eff }}=\Delta\left(\Delta / \omega_{\mathrm{c}}\right)^{K /(1-K)}$. The constant $c=$ $\left(4 \pi K e^{\bar{\gamma}}\right)^{K /(1-K)}$ is close to unity for $K \ll 1$.

In this paper, we focus on strong modification of the density of states caused by dissipation. The density of 


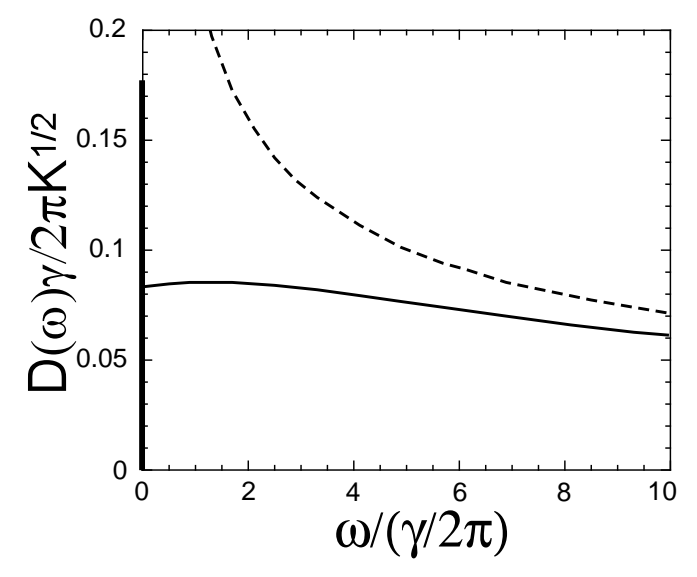

Fig. 2. The density of low-energy states $D(\omega)$ in the continuum limit for the dissipative case (the solid curve) and the dissipationless case (the dashed curve). The density of states for the dissipative case has a delta function at $\omega=0$, which is shown schematically by the thick line.

low-energy states is obtained from the low-temperature expansion of the partition function as 15

$$
D(\omega)=\sqrt{K} \delta(\omega)+\frac{1}{12 \sqrt{K} W}+\mathcal{O}(\omega)
$$

where $W=2 c \Delta_{\text {eff }}$ is the effective bandwidth, and the energy is shifted so that the ground-state energy becomes zero. This shape of $D(\omega)$ is quite different from the one in the dissipationless case where $D(\omega) \propto \omega^{-1 / 2}$.

We can obtain the whole shape of $D(\omega)$ analytically in the continuum limit: $K \rightarrow 0$ and $\Delta \rightarrow \infty$ with $K \Delta$ fixed. In this limit, the particle can be treated as a dissipative Brownian particle with a damping coefficient $\bar{n} 4 \pi K \Delta$, which is the only energy scale of the system 15, 16 In the continuum limit, the integral (13) is determined by the contribution around $\theta=0$, and can be evaluated by the saddle point method. Thus, the partition function is calculated as

$$
\begin{aligned}
\log Z & =2 \hbar \beta \Delta+\frac{1}{2} \log (2 \pi K)+\log \Gamma\left(\frac{\hbar \beta \gamma}{2 \pi}+1\right) \\
& -\left(\frac{\hbar \beta \gamma}{2 \pi}+\frac{1}{2}\right) \log \frac{\hbar \beta \gamma}{2 \pi} .
\end{aligned}
$$

From this expression, the density of states $D(\omega)$ can be obtained numerically by the inverse Laplace transform (17). The result takes the form

$$
D(\omega)=\sqrt{K} \delta(\omega)+\tilde{D}(\omega)
$$

The excited-state part $\tilde{D}(\omega)$ is shown in Fig. 2 by the solid curve, while the dissipationless case is shown by the dashed curve. The delta function at $\omega=0$ is also schematically drawn by the thick line in Fig. 2 .

If the dissipation effect appeared only in the suppres-

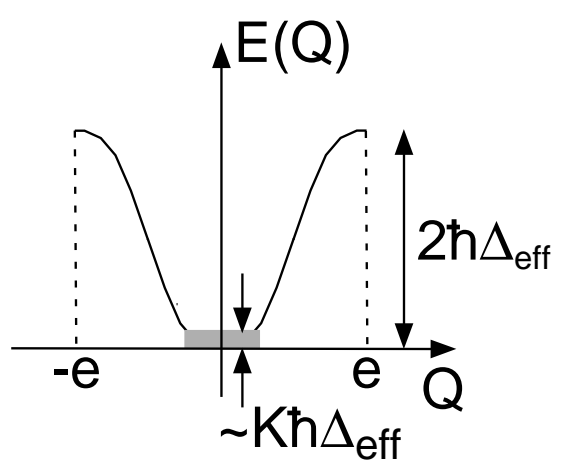

Fig. 3. The dispersion relation of the junction. Besides the energy scale of the band width $2 \hbar \Delta_{\text {eff }}$, there appears a new characteristic energy scale $\hbar \gamma \sim K \hbar \Delta_{\text {eff }}$ below which the dispersion is strongly modified in the gray region.

sion of the bare tunneling matrix $\Delta$ to $\Delta_{\text {eff }}$, the band dispersion would be given as

$$
E(Q)=2 \hbar \Delta_{\mathrm{eff}}(1-\cos (\pi Q / e)) .
$$

This dispersion, however, does not reproduce the present result of $D(\omega)$. In particular, the delta function in $D(\omega)$ at $\omega=0$ indicates that the low-energy states are degenerate around the band bottom. The qualitative feature of the dispersion is shown in Fig. 3, where the dispersion relation of relevant excitation is strongly modified in the gray region $\left(E<K \hbar \Delta_{\text {eff }}\right)$. For the moment, it is not yet confirmed whether this dispersion is directly related to the single particle dispersion relation because singleparticle Green's function has not been obtained yet because of difficulty in the analytical calculation even for $K \ll 1$.

As seen in Fig. 3, the dissipative system of $K \ll 1$ has two different energy scales: the bandwidth $\hbar \Delta_{\text {eff }}$ and the damping energy $\hbar \gamma \sim K \hbar \Delta_{\text {eff }}$. These two energy scales appear also in the $I-V$ characteristics as shown in the following subsections. Note that the existence of these two energy scales is not an artifact of the Debye-Hückel approximation. Actually, the nontrivial energy scale $\hbar \gamma$ appears in the continuum limit without the Debye-Hückel approximation by mapping the model to the Brownian motion problem, and the partition function (28) can be reproduced except for the divergent part $\log K$. (See also Appendix.)

As seen in (27), the weight of the delta function in $D(\omega)$ increases as $\sqrt{K}$ for $K \ll 1$. Hence, we expect that the macroscopic degeneracy at $\omega=0$ persists for general values of $K$, and that the weight of the delta function in $D(\omega)$ increases as $K$ is enlarged. This expectation gives a new aspect on the superconductor-insulator transition at $K=1$. As $K$ approaches unity, the effective bandwidth $\hbar \Delta_{\text {eff }}=\hbar \Delta\left(\Delta / \omega_{c}\right)^{K /(1-K)}$ is suppressed to be zero, and all the states become degenerate. Our result shows that a partial degeneracy remains 'even for $K<1$ '. 


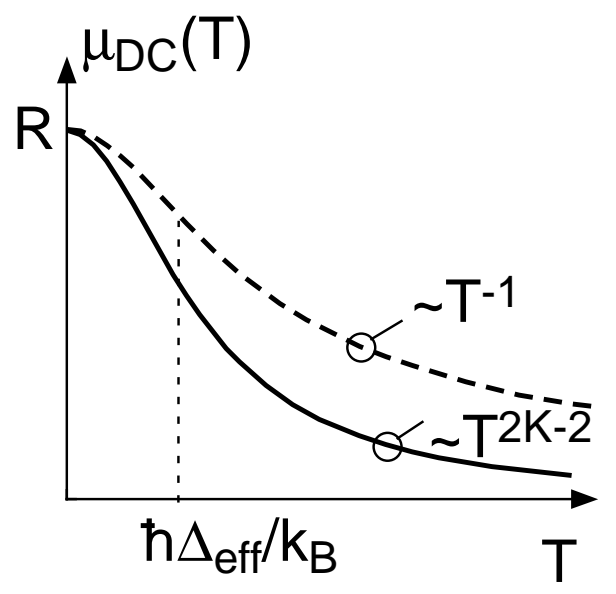

Fig. 4. The temperature-dependences of the DC mobility $\mu_{\mathrm{DC}}(T)$ are shown schematically by the solid curve for $K \ll 1$, and by the dashed curve for $K=1 / 2$.

This degeneracy in the density of states may be observed by the time-evolution measurement of the voltage, when the external current is large enough to cause the Bloch oscillation. If we consider a simplified model as discussed in Sec. 3.5, the flat dispersion generates no voltage. Hence, the time-dependence of the voltage may show the region of zero voltage for some interval.

\subsection{Linear DC responses}

The low- and high-temperature behavior of the DC mobility $\mu_{\mathrm{DC}}(T)$ can be obtained within the linear response theory for the weak damping region $K \ll 1$ as 15

$$
\mu_{\mathrm{DC}}(T)=\left\{\begin{array}{c}
R\left(1-\frac{1}{8}\left(\frac{k_{\mathrm{B}} T}{c \hbar \Delta_{\mathrm{eff}}}\right)^{2}+\mathcal{O}\left(T^{4}\right)\right), \\
\left(k_{\mathrm{B}} T \ll \hbar \Delta_{\mathrm{eff}}\right), \\
8 \pi^{2} R\left(\frac{\hbar \Delta_{\mathrm{eff}}}{2 \pi k_{\mathrm{B}} T}\right)^{2-2 K}, \\
\left(k_{\mathrm{B}} T \gg \hbar \Delta_{\mathrm{eff}}\right) .
\end{array}\right.
$$

The qualitative features of $\mu_{\mathrm{DC}}(T)$ for $K \ll 1$ obtained by connecting high- and low-temperature behavior are shown in Fig. 4 by the solid curve. At high temperatures, the mobility increases as the temperature decreases, while at low temperatures, the mobility saturates to $R$. The crossover temperature appears at $T \sim \hbar \Delta_{\text {eff }} / k_{\mathrm{B}}$.

We expect that this feature of $\mu_{\mathrm{DC}}(T)$ is universal for $0<K<1$. In fact, the feature of (31) is valid for the special case $K=1 / 2$, which allows analytical treatment.13) In this case, $\mu_{\mathrm{DC}}(T)$ is calculated exactly as

$$
\mu_{\mathrm{DC}}(T)=R \frac{\hbar \Delta_{\text {eff }}}{2 k_{\mathrm{B}} T} \psi^{\prime}\left(\hbar \Delta_{\mathrm{eff}} / 2 k_{\mathrm{B}} T+1 / 2\right),
$$

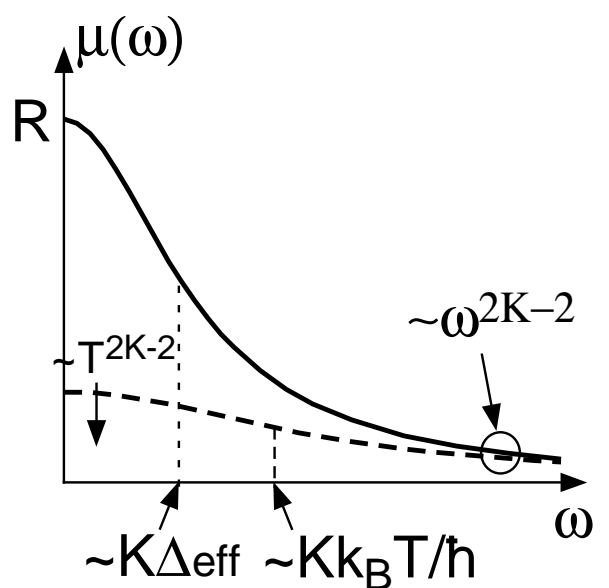

Fig. 5. The optical mobility $\mu(\omega ; T)$ for $K \ll 1$. The zerotemperature result is shown by the solid curve, and the hightemperature result by the dashed curve.

$$
=\left\{\begin{array}{l}
R\left(1-\frac{1}{3}\left(\frac{k_{\mathrm{B}} T}{\hbar \Delta_{\mathrm{eff}}}\right)^{2}\right), \\
\left(k_{\mathrm{B}} T \ll \hbar \Delta_{\mathrm{eff}}\right), \\
R \frac{\pi^{2} \hbar \Delta_{\mathrm{eff}}}{4 k_{\mathrm{B}} T}, \\
\left(k_{\mathrm{B}} T \gg \hbar \Delta_{\mathrm{eff}}\right),
\end{array}\right.
$$

where $\psi^{\prime}(x)$ is the digamma function. The qualitative behavior of $\mu_{\mathrm{DC}}(T)$ is shown in Fig. 4 by the dashed curve. This result of the $K=1 / 2$ case is consistent with our result (31).

These behaviors of $\mu_{\mathrm{DC}}(T)$ have been interpreted physically as follows At high temperatures, the phase $\phi$ slips by $2 \pi$ thermally. This phase slip occurs incoherently with a temperature-dependent tunneling rate determined essentially by the incoherent hopping in the two-level systems. As the temperature decreases, the phase-slip picture becomes inadequate, and the quantum coherence of the system appears. The wave function begins to extend in the $\phi$ space. Because of the large phase fluctuation, the Josephson junction behaves as an insulator, where Cooper pairs cannot tunnel through the junction. In this situation, the whole part of the current flows through the shunted resistance $R$, and the voltage follows the ohmic law $V=I R$, which corresponds to $\mu_{\mathrm{DC}}=R$.

\subsection{Linear optical responses}

In this subsection, we calculate the optical mobility $\mu(\omega ; T)$ within the linear response theory, which can be interpreted as the optical responses of the Josephson junctions.

At zero temperature, the optical mobility can be calculated analytically for $K \ll 1$ at any frequency, and the 
details of the results have been given in Ref. 15. Here, we discuss qualitative features of $\mu(\omega)$ from the analytical forms of the limiting regions:

$$
\mu(\omega ; T=0)= \begin{cases}\frac{R e^{-2 K \bar{\gamma}}}{2 \Gamma(2 K+1)}\left(\frac{\gamma}{\omega}\right)^{2-2 K}, & \left(\omega \gg \omega^{*}\right), \\ R \frac{\gamma^{2}}{\gamma^{2}+\omega^{2}}, & \left(\omega \ll \omega^{*}\right),\end{cases}
$$

where $\bar{\gamma}$ is the Euler constant. The qualitative features of $\mu(\omega)$ are shown in Fig. 5 by the solid curve. The damping frequency is given as $\gamma=4 \pi K c \Delta_{\text {eff }}$. Thus, the characteristic energy scale in $\mu(\omega)$ appears at $\hbar \gamma \sim K \hbar \Delta_{\text {eff }}$, which is the same order as the energy scale observed in the modification of the dispersion. The crossover frequency $\omega^{*}$ in (34) is evaluated as $\omega^{*} \sim 2^{1 / K} \gamma$. When we take $K \rightarrow 0$ and $\Delta \rightarrow \infty$ with $K \Delta$ fixed, the optical responses take exact Lorentzian forms $\left(\omega^{*} \rightarrow \infty\right)$, which are obtained also by considering the Brownian motion. 15 )

As $K$ increases, it is expected that the crossover frequency $\omega^{*}$ in (34) decreases, and the deviation from Lorentzian form becomes clear. At the same time, the effective hopping amplitude $\Delta_{\text {eff }}$ is suppressed. Particularly, in the limit $K \rightarrow 1$, the amplitude $\Delta_{\text {eff }}$ vanishes and the characteristic frequency $\gamma \sim K \Delta_{\text {eff }}$ in (34) also vanishes. For $K>1$, the dissipative particle moves only by the incoherent hopping, and the optical mobility takes a different form 11 )

$$
\mu(\omega ; T=0)=R \frac{8 \pi^{2} K^{2} \Delta^{2}}{\Gamma(2 K+1) \omega_{\mathrm{c}}^{2}}\left(\frac{\omega}{\omega_{\mathrm{c}}}\right)^{2 K-2} .
$$

The finite-temperature calculation of $\mu(\omega ; T)$ is difficult even for $K \ll 1$. For a qualitative discussion, however, it is sufficient to consider at high-temperature $k_{\mathrm{B}} T \gg \hbar \Delta_{\text {eff }}$, where the optical mobility is calculated for arbitrary values of $K$ by assuming the incoherent hopping of the dissipative particle. The optical mobility is obtained for $0<K<1$ as 11 )

$$
\begin{aligned}
\mu(\omega ; T)= & R 4 \pi K \Delta_{\mathrm{eff}}\left(\frac{\hbar \Delta_{\mathrm{eff}}}{2 \pi k_{\mathrm{B}} T}\right)^{2-2 K} \\
& \times \frac{\left|\Gamma\left(K+\mathrm{i} \hbar \omega / 2 \pi k_{\mathrm{B}} T\right)\right|^{2}}{\Gamma(2 K)} \frac{\sinh \hbar \omega / 2 k_{\mathrm{B}} T}{\omega} \\
& =\left\{\begin{array}{c}
R \frac{8 \pi^{2} K^{2}}{\Gamma(2 K+1)}\left(\frac{\Delta_{\mathrm{eff}}}{\omega}\right)^{2-2 K} \\
\left(\omega \gg K k_{\mathrm{B}} T / \hbar\right) \\
R \frac{8 \pi^{2} K^{2} \Gamma(K)^{2}}{\Gamma(2 K+1)}\left(\frac{\hbar \Delta_{\mathrm{eff}}}{2 \pi k_{\mathrm{B}} T}\right)^{2-2 K} \\
\left(\omega \ll K k_{\mathrm{B}} T / \hbar\right)
\end{array}\right.
\end{aligned}
$$

The qualitative behavior is shown by the dashed curve in Fig. 5. The high-frequency side is temperatureindependent, and agrees with the zero-temperature result in the weak damping region $K \ll 1$. This shows that the high frequency side is well described by the incoherent hopping of the dissipative particle even at zero

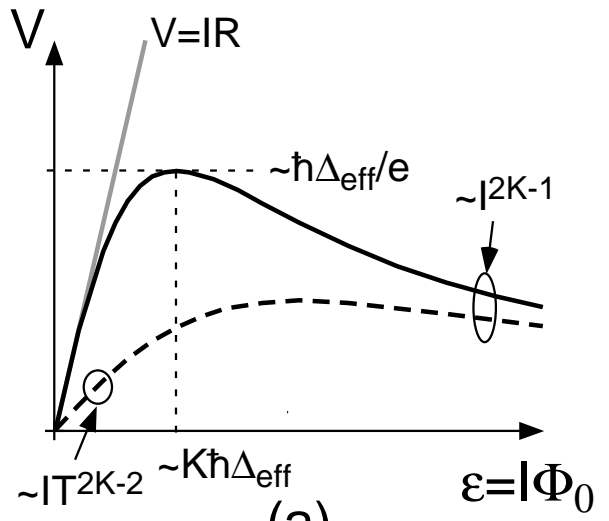

(a)

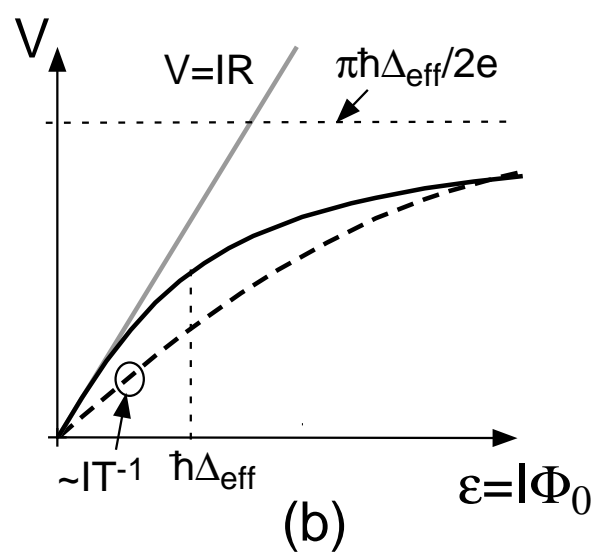

Fig. 6. (a) The obtained $I-V$ characteristics for $0<K<1$, and (b) the exact solution at $K=1 / 2$. The zero-temperature results are shown by the solid curves, and the high-temperature results by the dashed curves. The Ohmic responses $V=I R$ are also shown by gray lines for references.

temperature. The low-frequency side is temperaturedependent, and behaves as $T^{2 K-2}$. The crossover frequency is given as $\omega \sim K k_{\mathrm{B}} T / \hbar$.

When the temperature decreases, the low-frequency temperature-dependent part in (36) becomes inadequate below a crossover temperature. This crossover temperature is estimated for $K \ll 1$ as $T \sim \hbar \Delta_{\text {eff }} / k_{\mathrm{B}}$ where the characteristic frequency in (36) takes the same order $\left(\gamma=4 \pi K \Delta_{\text {eff }} \sim K k_{\mathrm{B}} T / \hbar\right)$ as that seen in (34). This result is consistent with that of the DC mobility, where the crossover temperature is given also as $T \sim \hbar \Delta_{\text {eff }} / k_{\mathrm{B}}$.

\section{$3.4 \quad$ Nonlinear responses}

Theoretically, it is a difficult task to study nonlinear responses beyond the linear response theory, because nonequilibrium states must be treated properly. In this paper, general properties of the $I-V$ characteristics are inferred from the results obtained in some limits.

At zero temperature, the nonlinear mobility $\mu_{\mathrm{NL}}=$ 
$V / I$ can be calculated for large $I$ as 11 )

$$
\mu_{\mathrm{NL}}(\varepsilon ; T=0)=R \frac{8 \pi^{2} K^{2}}{\Gamma(2 K+1)}\left(\frac{\hbar \Delta_{\mathrm{eff}}}{\varepsilon}\right)^{2-2 K},
$$

where $\varepsilon=I \Phi_{0}$ is the energy difference between neighboring wells in the periodic potential $U(q)$. For large $I$, the voltage $V=\mu_{\mathrm{NL}} I$ is proportional to $I^{2 K-1}$. On the other hand for small $I$, the mobility $\mu_{\mathrm{NL}}$ is expected to be $R$ for $0<K<1$ from the renormalization group analysis.5) By connecting these two limiting cases smoothly, the $I-V$ characteristics are derived as in Fig. 6 (a) by the solid curve.

The crossover current is determined by the cross point of the results of the two limiting cases. Particularly for $K \ll 1$, the crossover current is $\varepsilon \sim K \hbar \Delta_{\text {eff }}$, while the characteristic voltage is $V \sim I R \sim \hbar \Delta_{\text {eff }} / e$. Thus, also in the $I-V$ characteristics, two different energy scales, $\hbar \Delta_{\text {eff }}$ and $K \hbar \Delta_{\text {eff }}$ appear clearly.

The high-temperature limit $k_{\mathrm{B}} T \gg \hbar \Delta_{\text {eff }}$ can also be calculated analytically for $0<K<1$ as

$$
\begin{aligned}
\mu_{\mathrm{NL}}(\varepsilon ; T)= & R 4 \pi K \hbar \Delta_{\mathrm{eff}}\left(\frac{\hbar \Delta_{\mathrm{eff}}}{2 \pi k_{\mathrm{B}} T}\right)^{1-2 K} \\
& \times \frac{\left|\Gamma\left(K+\mathrm{i} \varepsilon / 2 \pi k_{\mathrm{B}} T\right)\right|^{2}}{\Gamma(2 K)} \frac{\sinh \left(\varepsilon / k_{\mathrm{B}} T\right)}{\varepsilon} \\
= & \left\{\begin{array}{c}
R \frac{8 \pi^{2} K^{2}}{\Gamma(2 K+1)}\left(\frac{\hbar \Delta_{\mathrm{eff}}}{\varepsilon}\right)^{2-2 K}, \\
\left(\varepsilon \gg k_{\mathrm{B}} T\right), \\
R \frac{8 \pi^{2} K^{2} \Gamma(K)^{2}}{\Gamma(2 K+1)}\left(\frac{\hbar \Delta_{\mathrm{eff}}}{2 \pi k_{\mathrm{B}} T}\right)^{2-2 K} \\
\left(\varepsilon \ll k_{\mathrm{B}} T\right) .
\end{array}\right.
\end{aligned}
$$

The schematic $I-V$ characteristics are shown also in Fig. 6 (a) by the dashed curve. At large current $(\varepsilon=$ $\left.\Phi_{0} I \gg k_{\mathrm{B}} T\right)$, the mobility is temperature-independent, and behaves as $I^{2 K-2}$, while at small current $(\varepsilon \ll$ $\left.k_{\mathrm{B}} T\right)$ it becomes temperature-dependent. Note that in this regime the nonlinear DC mobility is obtained from the linear optical conductivity as $\mu_{\mathrm{NL}}(\varepsilon ; T)=\mu(\omega \rightarrow$ $\varepsilon / \hbar ; T)$.

We expect that the obtained $I-V$ characteristics are valid for any values of $K$. This conjecture is easily checked by considering the exact solution of the $K=1 / 2$ case given by 13 )

$$
\begin{aligned}
\mu_{\mathrm{NL}}(\varepsilon ; T)= & R \frac{4 \pi \hbar \Delta_{\text {eff }}}{\varepsilon} \operatorname{Im} \psi\left(\frac{1}{2}+\frac{2 \hbar \Delta_{\text {eff }}}{k_{\mathrm{B}} T}+\mathrm{i} \frac{\varepsilon}{2 \pi k_{\mathrm{B}} T}\right) \\
= & \left\{\begin{array}{l}
R \frac{2 \pi^{2} \hbar \Delta_{\text {eff }}}{\varepsilon} \tanh \left(\frac{\varepsilon}{2 k_{\mathrm{B}} T}\right), \\
\left(k_{\mathrm{B}} T \gg \hbar \Delta_{\text {eff }}\right), \\
R \frac{4 \pi \hbar \Delta_{\text {eff }}}{\varepsilon} \arctan \left(\frac{\varepsilon}{4 \pi \hbar \Delta_{\text {eff }}}\right),
\end{array}\right.
\end{aligned}
$$

The $I$ - $V$ characteristics in the $K=1 / 2$ case is shown in
Fig. 6 (b) for $k_{\mathrm{B}} T \ll \hbar \Delta_{\text {eff }}$ by the solid curve, and for $k_{\mathrm{B}} T \gg \hbar \Delta_{\text {eff }}$ by the dashed curve. This $I-V$ characteristics are consistent with the ones obtained in Fig. 6 (a) when we take $K=1 / 2$ in our present result.

\subsection{Discussion based on a simplified model}

The above results can be understood intuitively in terms of the Coulomb blockade and the Bloch oscillation by a simple analysis based on the equation of motion

$$
\dot{Q}+\frac{V_{\mathrm{x}}}{R}=I,
$$

which is a simplified version of the one analyzed in Ref. 3 in detail. Here, $Q$ and $I$ are the charge in the junction and the external current, respectively. The second term in (42) denotes the normal current through the shunted resistance. The voltage is calculated by $V_{\mathrm{x}}=\mathrm{d} E(Q) / \mathrm{d} Q$, where $E(Q)$ is the dispersion relation given by (30). The kinetic energy of the particle originates from the Coulomb energy, and $C_{\text {eff }}=e^{2} / \hbar \Delta_{\text {eff }}$ plays a role of an effective capacitance. The Josephson effects appear from the fact that the pseudo-momentum $Q$ is folded into the region $-e \leq Q \leq e$. For the tight-binding limit $E_{\mathrm{J}} / E_{\mathrm{C}} \gg 1$, it is allowed to focus only on the lowest band. We note that (42) is justified only when $Q$ is a classical variable and quantum fluctuations are negligible.

By solving the equation (42), we find that the junction properties change at a critical current $I^{*}=2 \pi \hbar \Delta_{\text {eff }} / e R$. For $I<I^{*}$, the charge converges to a finite value $(\dot{Q}=$ $0)$. In this regime, a Cooper pair cannot tunnel through the junction, because it costs additional Coulomb energy for the Cooper-pair to tunnel. This behavior is easily understood as the Coulomb blockade phenomena.

For the opposite case $I>I^{*}$, the charge $Q$ continues to increase until $Q$ becomes $e$, and then the value of $Q$ jumps to $-e$ by tunneling of a Cooper pair. This process iterates permanently. In this regime, there appears voltage oscillation with the frequency $f=I / 2 e$ known as the Bloch oscillation 3 )

From the simplified model (42), the voltage averaged over the period $T=1 / f$ is given as

$$
V=\int_{0}^{T} \frac{\mathrm{d} t}{T} V_{\mathrm{x}}
$$

and is calculated as a function of $I$. The result is shown in Fig. 7. For $I<I^{*}$, the $I-V$ characteristics are expressed exactly as $V=I R$, because no Cooper pair can tunnel through the junction by the Coulomb blockade. For $I>I^{*}$, the junction retains superconductivity by opening the channel of the Cooper-pair tunneling, and the voltage is reduced. The whole shape of this $I$ - $V$ characteristics is similar with the one obtained in the previous subsection. Particularly, the characteristic voltage $V^{*}$ and current $\varepsilon^{*}=I^{*} \Phi_{0}$ are consistent with 


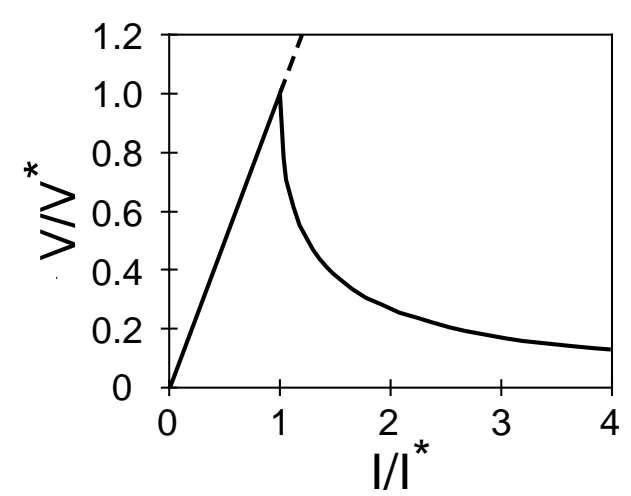

Fig. 7. The $I-V$ characteristics calculated from the simplified model (42). The current and voltage is normalized by $I^{*}=$ $2 \pi \hbar \Delta_{\text {eff }} / e R$ and $V^{*}=2 \pi \hbar \Delta_{\text {eff }} / e$, respectively. The dashed line denotes the Ohmic response $V=I R$.

the one obtained in the last subsection $\left(V^{*} \sim \hbar \Delta_{\text {eff }} / e\right.$, $\left.\varepsilon^{*} \sim K \hbar \Delta_{\text {eff }}\right)$. Thus, the two energy scales $\hbar \Delta_{\text {eff }}$ and $K \hbar \Delta_{\text {eff }}$ appear also in this simplified model.

We, however, should note that the simplified model does not reproduce correct results at a quantitative level due to the absence of quantum fluctuations of $Q$. The differences can be pointed out as follows: (1) the asymptotic behavior for $I \gg I^{*}$ of the simplified model is given by $V \propto 1 / I$, while the exact calculation gives $V \propto I^{2 K-1}$, (2) the exact solution for $K=1 / 2$ suggests that there is no cusp in the $I-V$ characteristics for any $K$ in contrast to the result obtained from the simplified model, and (3) the exact solution for $K=1 / 2$ also clarify that even for small $I$ the superconductivity of the junction is partially retained as seen in the deviation of the $I-V$ characteristics deviate from the $V=I R$ line. Thus, it is clear that the simplified model cannot be used for the quantitative discussion.

\section{§4. Relation to the Kondo problem}

In this section, the dissipative tight-binding model is discussed in relation to the Kondo problem. Although this discussion is not helpful in analyzing unsolved problems about the dissipative tight-binding model, it provides us with a new perspective, which may contribute to a better understanding of the junction properties.

First, we consider dissipative systems with a doublewell potential. When the systems are truncated to twolevel systems, the Hamiltonian is obtained as

$H=-\frac{\hbar \Delta}{2} \sigma_{x}+\sum_{j}\left\{\frac{p_{j}^{2}}{2 m_{j}}+\frac{m_{j} \omega_{j}^{2}}{2}\left(x_{j}-\frac{c_{j}}{m_{j} \omega_{j}^{2}} \frac{a}{2} \sigma_{z}\right)^{2}\right\}$,

where $\sigma_{x}$ and $\sigma_{z}$ are the Pauli matrices. This model has been studied as the simplest model describing destruction of quantum coherence by surrounding medium.

In this paper, the dissipative 'multi-level' system is studied. One may regard this model as an extension of the dissipative 'two-level' system. There, however, exists a critical difference between these two models. In dissipative two-level systems, the dissipation is introduced to the 'gaped' system with a gap $\hbar \Delta$, while in multilevel systems, to the system with a 'gapless' band with a bandwidth $2 \hbar \Delta$. Though these two models show essentially the same behavior at high temperature, the low-temperature properties are quite different. Actually, density of states for dissipative two-level systems has no characteristic feature at the energyscale $K \hbar \Delta_{\text {eff }}$ in contrast with the multi-level systems.17) The energy scale $K \hbar \Delta_{\text {eff }}$ appears in the dissipative two-level systems only as a characteristic relaxation time in the correlation function and ip the evolution of the expectation value $P(t)=\left\langle\sigma_{z}(t)\right\rangle .18$

Even in this dissipative two-level model, it is a nontrivial task to study the system properties such as thermodynamics and real-time correlation functions. Actually, it has been proven that the low-energy behavior of this dissipative two-level model is equivalent to the Kondo model with anisotropic exchange couplings: 19

$$
H_{\mathrm{K}}=\sum_{k, \sigma} \varepsilon_{k} c_{k, \sigma}^{\dagger} c_{k, \sigma}+J_{\|} S_{z} s_{z}+J_{\perp}\left(S_{x} s_{x}+S_{y} s_{y}\right) \text {. }
$$

Here, $c_{k, \sigma}\left(c_{k, \sigma}^{\dagger}\right)$ is an annihilation (creation) operator of a conduction electron, $S_{i}$ is the local spin operator, and $s_{i}$ is the spin operator of the conduction electrons at the impurity site. The equivalence between the Kondo model and the dissipative two-level system is obtained by identifying the coupling constants $J_{\|}$and $J_{\perp}$ with20

$$
\begin{gathered}
\frac{\Delta}{\omega_{c}}=\rho J_{\perp} \cos ^{2} \frac{\pi \rho J_{\|}}{4}, \\
K=\left(1-\frac{\rho J_{\|}}{2}\right)^{2},
\end{gathered}
$$

where $\rho$ is the density of states at the Fermi surface. Unfortunately, the weak damping region $K \ll 1$ cannot be related to the Kondo problem exactly, since the above relations become valid only in the limit $\left.\rho J_{\|}, \rho J_{\perp} \ll 1.20\right)$

The properties of the dissipative two-level model can be understood in terms of the Kondo problem as follows.2)

In the dissipative two-level systems, the effective hopping amplitude $\Delta_{\text {eff }}$ determines the energy scale of the system. At high temperatures $k_{\mathrm{B}} T \gg \hbar \Delta_{\text {eff }}$, the coherence of the system is destroyed by dissipation, and the energy levels become degenerate. The particle is localized at one well, and hops to the other incoherently. At low temperatures $k_{\mathrm{B}} T \ll \hbar \Delta_{\text {eff }}$, the coherence of the system is retained, and the energy splitting is given by $\hbar \Delta_{\text {eff }}$.

In the (anisotropic) Kondo model, the Kondo temperature $T_{\mathrm{K}}$ determines the energy scale of the system. At high temperatures $T \gg T_{\mathrm{K}}$, the local impurity spin fluc- 
tuates thermally, and the magnetic susceptibility of the local spin $\chi$ follows the Curie law. At low temperatures $T \ll T_{\mathrm{K}}$, the local impurity spin forms a singlet state coupled with conduction electrons, and $\chi$ becomes independent of the temperature.

In the both cases, at a characteristic temperature $\left(\hbar \Delta_{\text {eff }}\right.$ or $\left.k_{\mathrm{B}} T_{\mathrm{K}}\right)$, the behavior of the system changes drastically. This common feature actually comes from the equivalence of these two models.19.20

From this viewpoint, let us come back to the results obtained in the previous section. In the dissipative multilevel model, there are two different energy scales, which are seen clearly in the calculation for $K \ll 1$. One is the effective bandwidth $2 \hbar \Delta_{\text {eff }}$, which can be related to the Kondo temperature $T_{\mathrm{K}}$. This energy scale appears as a crossover temperature in the DC mobility, and as a characteristic voltage $V \sim \hbar \Delta_{\text {eff }} / 2 e$. The other is the new energy scale $\hbar \gamma \sim K \hbar \Delta_{\text {eff }}$, which can be seen in the density of states $D(\omega)$ (see Fig. 3), and in the optical mobility $\mu(\omega)$. This energy scale is characteristic of the multi-level model, in which the dissipation is introduced to a gapless system. This situation cannot be realized in the Kondo problem.

In this sense, the resistance-shunted Josephson junction system offers a new type of nontrivial stronglycorrelated problems. Though it has some similarity, it also has an essential difference from the Kondo problem. If properly designed, Josephson devices may show such a novel phenomenon originating from strongly correlation effects.

\section{$\S 5$. Summary}

In this paper, thermodynamics and linear responses for the limiting region $\left(K \ll 1\right.$ and $\left.E_{\mathrm{J}} / E_{\mathrm{C}} \gg 1\right)$ have been studied analytically for all temperatures. The nonlinear $I-V$ characteristics are also discussed from the available results.

The density of low-energy states $\left(\omega<K \Delta_{\text {eff }}\right)$ is strongly modified. This modification indicates that the dispersion relation of relevant excitations is strongly altered at the band bottom. The DC mobility $\mu_{\mathrm{DC}}(T)=$ $V / I$ behaves as $T^{2 K-2}$ at high temperatures, while it saturates to $R$ below a crossover temperature $T \sim$ $\hbar \Delta_{\text {eff }} / k_{\mathrm{B}}$. At zero temperature, the optical responses follow a Lorentzian form with a width $\gamma=4 \pi K \Delta_{\text {eff }}$ at low frequencies, while they behave as $\omega^{2 K-2}$ at high frequencies. Above a crossover temperature $T \sim \hbar \Delta_{\text {eff }} / k_{\mathrm{B}}$, the optical mobility becomes temperature-dependent on the low-frequency side. The nonlinear $I-V$ characteristics for $0<K<1$ change at a crossover current $I^{*} \sim K \hbar \Delta_{\text {eff }} / \Phi_{0}$. For $I<I^{*}$, the system behaves like an insulator following the ohmic law $V=I R$, while for $I>I^{*}$ the junction retains the superconductivity, and behaves as $V \sim I^{2 K-1}$. These features are reproduced qualitatively by a simplified model. Among others, this system shows two different energy scales. One is the effective bandwidth $\hbar \Delta_{\text {eff }}$, and the other is $K \hbar \Delta_{\text {eff }}$. The latter energy scale appears as the consequence of strong coupling to the dissipation and is seen in the density of states, optical mobility and $I-V$ characteristics. The Josephson devices can be recognized as novel stronglycorrelated systems.

In this paper, the junction properties have been studied mainly in the weak damping region $K \ll 1$ and the tight-binding limit $E_{\mathrm{J}} / E_{\mathrm{C}} \gg 1$. These two conditions can be realized easily in experimental situations. In the tight-binding region, however, the effective bandwidth $\hbar \Delta_{\text {eff }}$ must be small (typically $\hbar \Delta_{\text {eff }} / k_{\mathrm{B}}<1[\mathrm{mK}]$ ). Then it becomes difficult to lower the temperature below $\hbar \Delta_{\text {eff }} / k_{\mathrm{B}}$. For experimental feasibility, the region $E_{\mathrm{J}} \sim E_{\mathrm{C}}$ would be more fruitful, where the qualitatively similar feature predicted in this paper can be observed. Actually, also in the opposite limit $E_{\mathrm{J}} / E_{\mathrm{C}} \ll 1$, two different energy scales appear: the effective band width $\Delta=e^{2} / 2 C$ determines the characteristic temperature of the linear mobility, while the damping energy $\gamma \sim K \Delta$ determines the characteristic current $\varepsilon=I \Phi_{0}$ of the nonlinear mobility.5) The analyses in this paper may contribute to the understanding of quantum effects in the Josephson junctions also in those parameter regions.

The authors thank G. Schön for useful suggestion. T.K. is supported by Research Fellowship of Japan Society for the Promotion of Science for Young Scientists. This study was supported by a Grant-in-Aid for Scientific Research from the Japanese Ministry of Education, Science, Sports and Culture. This study was also supported by a JSPS project 'Research for the Future' (JSPS-RFTF 97P01103).

\section{Appendix: Validity of the Debye-Hückel Ap- proximation}

The concept of this approximation has first been considered by Debye and Hückel.21) Later, Mayer has shown that the Debye-Hückel theory can be reproduced from the ring approximation in the classical cluster expansion.22) Since then, the corrections to the Debye-Hückel approximation have been studied for the problem of interacting plasma.

Let us consider the 3D classical Coulomb gas with the particle density $n$. In the Debye-Hückel approximation, the two-body distribution function is calculated as

$$
\rho_{2}(r)=n^{2} \exp \left(-\frac{\phi_{\mathrm{D}}(r)}{T}\right) .
$$

Here, $T$ is the temperature, and $\phi_{\mathrm{D}}(r)$ is the screened potential

$$
\phi_{\mathrm{D}}(r)=e^{2} \frac{e^{-\kappa_{\mathrm{D}} r}}{r},
$$


where $1 / \kappa_{\mathrm{D}}$ is the Debye screening length given by

$$
\kappa_{\mathrm{D}}=\left(\frac{4 \pi n e^{2}}{T}\right)^{1 / 2}
$$

Note that the two-body distribution function can be written in a simple form as

$$
\rho_{2}(r)=n^{2} \exp \left(-\varepsilon \frac{e^{-x}}{x}\right),
$$

where $x=\kappa_{\mathrm{D}} r$ is the dimensionless spatial coordinate, and $\varepsilon=e^{2} \kappa_{\mathrm{D}} / T$ is the plasma parameter. Beyond the ring approximation, it is possible to sum the graphs up to the order of $\varepsilon^{2}$ in the cluster expansion.23,24] The result is given as

$$
\rho_{2}(r)=n^{2} \exp \left(-\varepsilon \frac{e^{-x}}{x}-\varepsilon^{2} f(x)\right),
$$

where $f(x)$ is a complex function of $x$. From this result, if the series of $\varepsilon$ is convergent, then the ring approximation is justified for $\varepsilon \ll 1$. Also the results by the Monte Carlo simulation 25, 26) have supported Eq. (A.5. Thus, the Debye-Hückel approximation is thought to be valid in the weak coupling limit $\varepsilon \ll 1$ for the $3 \mathrm{D}$ classical Coulomb gas.

One may suspect that the Debye-Hëkel theory is not applicable to one-dimensional systems. Here, let us discuss the $1 \mathrm{D}$ classical Coulomb gas described by the Hamiltonian

$$
H=\frac{1}{2} \sum_{i} p_{i}^{2}-2 \pi e^{2} \sum_{i<j} \sigma_{i} \sigma_{j}\left|q_{i}-q_{j}\right|
$$

where $p_{j}, q_{j}$, and $\sigma_{j}= \pm 1$ are the momentum, position, and sign of the $j$-th charged particle. Among $2 N$ particles, $N$ particles are charged positively and $N$ neoatively. Fortunately, this model can be solved exactly.27 We take the dimensionless parameter as $\gamma=P / 2 \pi e^{2}$, where $P$ is the pressure. In this $1 \mathrm{D}$ model, the system behaves as ideal gas in the high-pressure limit $\gamma \rightarrow \infty$ on the contrary to the $3 \mathrm{D}$ case. The energy per particle $u$ is calculated for $\gamma \gg 1$ in the expansion form as

$$
u=\frac{T}{2}+\frac{T}{2}\left\{1+\frac{1}{(2 \gamma)^{1 / 2}}-\frac{1}{8 \gamma}+\frac{1}{128 \sqrt{2} \gamma^{3 / 2}}+\cdots\right\} .
$$

It can easily be checked that the Debye-Hückel approximation gives the exact result $(\mathrm{A} \cdot 7)$ up to the order of $1 / \gamma^{1 / 2}$. Thus, this approximation is also applicable to the $1 \mathrm{D}$ classical Coulomb gas for the weak coupling region $\gamma \gg 1$.

From the above discussions, the Debye-Hückel approximation is thought to be applicable to general classical plasma models in the condition

$$
1 / \kappa_{\mathrm{D}} \gg \bar{l}
$$

where $\bar{l}$ is the averaged particle distance. This condition is in fact reasonable from the physical viewpoint because the Debye-Hückel approximation, an effective field approach, is expected to become reliable when many particles are interacting each other within the screening radius. To clarify further the validity of the ring approximation, other methods such as Monte Carlo simulations would be helpful.

To study the dissipative tight-binding model, 1D classical particles interacting with the potential $\phi(\tau)$ in the $\tau$ direction have been considered. The potential is formulated as

$$
\begin{aligned}
& \phi(\tau)=\frac{1}{\hbar \beta} \sum_{\omega_{m}} \phi\left(\mathrm{i} \omega_{m}\right) e^{-\mathrm{i} \omega_{m} \tau}, \\
& \phi\left(\mathrm{i} \omega_{m}\right)=-\frac{2 \pi K}{\left|\omega_{m}\right|},
\end{aligned}
$$

for $0<\left|\omega_{m}\right| \ll \omega_{c}$, while the potential vanishes for $\omega_{m}>\omega_{\mathrm{c}}$. The condition (A.8) is evaluated for the dissipative tight-binding model as follows. In the DebyeHückel approximation, the screened potential $\varphi\left(\mathrm{i} \omega_{m}\right)$ is calculated as

$$
\varphi\left(\mathrm{i} \omega_{m}\right)=\frac{\phi\left(\mathrm{i} \omega_{m}\right)}{1-n \phi\left(\mathrm{i} \omega_{m}\right) / \hbar \beta} .
$$

The screening effects appears when the second term of the denominator in $(\mathrm{A} \cdot 11)$ is dominant. Hence, the Debye screening length $1 / \kappa_{\mathrm{D}}$ is determined from $n \phi\left(\omega_{m}=\right.$ $\left.\kappa_{D}\right) / \hbar \beta \sim 1$, and the condition $(\mathrm{A} \cdot 8$ ) corresponds to the weak-damping condition $K \ll 1$. Thus, we expect the ring approximation gives reliable results in the weakdamping region.

It can be proven that the Debye-Hückel theory reproduces correct results in the high temperature and/or strong damping region.15) The Debye-Hückel approximation also reproduces the zero-temperature mobility, which is obtained by the renormalization group analysis ast 4 ,

$$
\mu_{\mathrm{DC}}(T=0)=R \text {. }
$$

Further, in the continuum limit $(K \rightarrow 0, \Delta \rightarrow \infty$ with $\gamma=4 \pi K \Delta$ fixed), it can be proven that the DebyeHückel approximation gives corrects results for the optical mobility.15) Also for thermodynamics, it can be checked that the energy calculated in the Debye-Hückel approximation

$$
E=\text { const. }+\frac{\hbar \gamma}{2 \pi}\left[\log \left(\frac{\hbar \beta \gamma}{2 \pi}\right)-\psi\left(\frac{\hbar \beta \gamma}{2 \pi}\right)-\frac{\pi}{\hbar \beta \gamma}\right]
$$

agrees with the energy of a dissipative Brownian particle obtained in the continuum limit as 4 )

$$
\left\langle\frac{p^{2}}{2 M}\right\rangle=\frac{\hbar}{2 \pi} \int_{0}^{\infty} \mathrm{d} \omega \frac{\omega \gamma}{\omega^{2}+\gamma^{2}} \operatorname{coth}(\omega \hbar \beta / 2) .
$$

These provide further supports for the reliability of the Debye-Hückel theory in this problem. 
[1] A. O. Caldeira and A. J. Leggett: Ann. Phys. (N. Y.) 149 (1983) 374 [Errata; 153 (1984) 445].

[2] A. J. Leggett, S. Chakravarty, A. T. Dorsey, M. P. A. Fisher, A. Garg and W. Zwerger: Rev. Mod. Phys. 59 (1987) 1 [Errata; 67 (1995) 725].

[3] G. Schön and Z. D. Zaikin: Phys. Rep. 198 (1990) 237.

[4] U. Weiss: Quantum Dissipative Systems (World Scientific, Singapore, 1993).

[5] M. P. A. Fisher and W. Zwerger: Phys. Rev. B 32 (1985) 6190.

[6] R. Yagi, S. Kobayashi and Y. Ootsuka: J. Phys. Soc. Jpn. 66 (1997) 3722.

[7] J. S. Penttilä, Ü. Parts, P. J. Hakonen, M. A. Paalanen and E. B. Sonin: Phys. Rev. Lett. 82 (1999) 1004.

[8] U. Eckern and F. Pelzer: Europhys. Lett. 3 (1987) 131.

[9] W. Zwerger: Phys. Rev. B 35 (1987) 4737.

[10] S. E. Korshunov: Sov. Phys. JETP 66 (1987) 872.

[11] U. Weiss and H. Grabert: Phys. Lett. 108A (1985) 63.

[12] Y.-C. Chen, J. L. Lebowitz and C. Liverani: Phys. Rev. B 40 (1989) 4664.

[13] U. Weiss, M. Sassetti, T. Negele and M. Wollensak: Z. Phys. B 84 (1991) 471.

[14] M. Sassetti, M Milch and U. Weiss: Phys. Rev. 46 (1992) 4615.

[15] T. Kato and M. Imada: J. Phys. Soc. Jpn. 67 (1998) 2828.

[16] The density of states in the continuum limit has not been calculated in Ref. 15

[17] R. Görlich and U. Weiss: Phys. Rev. B 38 (1988) 5245.

[18] U. Weiss and M. Wollensak: Phys. Rev. Lett. 62 (1989) 1663.

[19] F. Guinea, V. Hakim, A. Muramatsu: Phys. Rev. B 32 (1985) 4410.

[20] G. Yuval and P. W. Anderson: Phys. Rev. B 1 (1970) 1522.

[21] P. Debye and G. Hückel: Physik. Z. 24 (1923) 305.

[22] J. Mayer: J. Chem. Phys. 18 (1950) 1426.

[23] A. Ishihara: Phys. Rev. 178 (1969) 412.

[24] A. Ishihara and M. Wadachi: Phys. Rev. 183 (1969) 312.

[25] W. L. Slattery, G. D. Doolen and H. E. DeWitt: Phys. Rev. A 21 (1980) 2087.

[26] W. L. Slattery, G. D. Doolen and H. E. DeWitt: Phys. Rev. A 26 (1982) 2255.

[27] A. Lenard: J. Math. Phys. 2 (1961) 682. 